\title{
Téoros
}

Revue de recherche en tourisme

\section{Le tourisme québécois a-t-il encore besoin de l'État ?}

\section{André Coupet}

Volume 9, numéro 1, mars 1990

Plans et politiques touristiques

URI : https://id.erudit.org/iderudit/1080289ar

DOI : https://doi.org/10.7202/1080289ar

Aller au sommaire du numéro

Éditeur(s)

Université du Québec à Montréal

ISSN

0712-8657 (imprimé)

1923-2705 (numérique)

Découvrir la revue

Citer cet article

Coupet, A. (1990). Le tourisme québécois a-t-il encore besoin de l’État? Téoros, 9(1), 23-28. https://doi.org/10.7202/1080289ar d'utilisation que vous pouvez consulter en ligne.

https://apropos.erudit.org/fr/usagers/politique-dutilisation/ 


\section{Le tourisme québécois a-t-il encore besoin de l'Etat?}

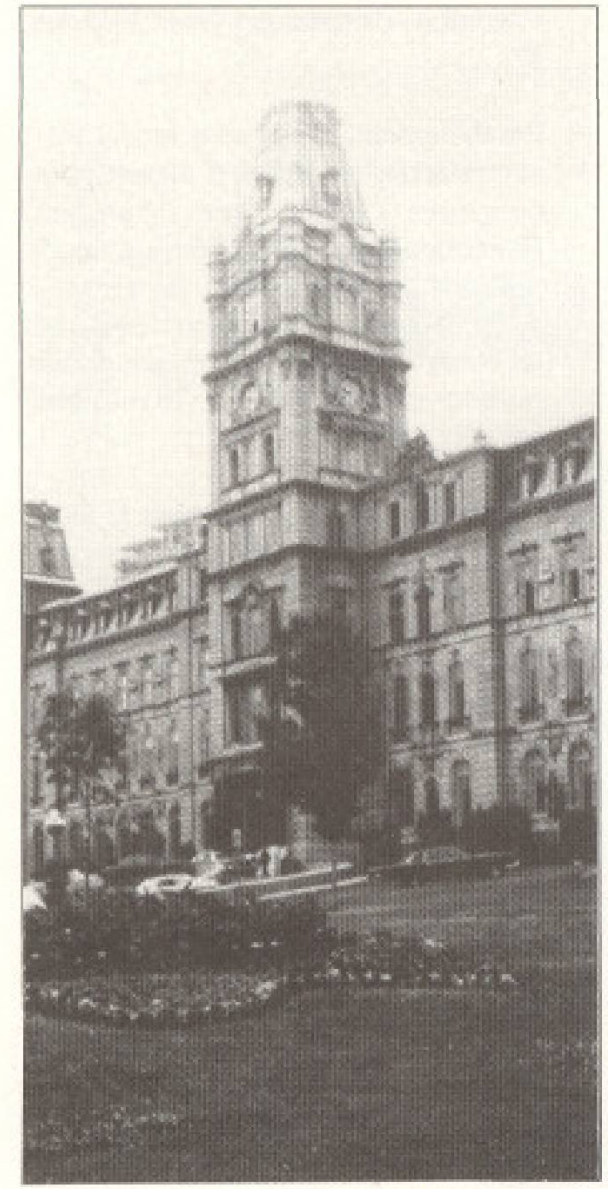

Monsieur André Coupet est directeur du Service de consultation en marketing et en planification strategique chez Samson BelairDeloitte \& Touche.
Le gouvernement du Québec a beaucoup investi dans l'industrie touristique même si le secteur privé a toujours été et demeure 1'agent économique dominant non seulement au niveau des recettes mais également au niveau des investissements.

Au début des années '90, en tourisme comme dans d'autres secteurs, il devient urgent de se poser la question si le rôle de l'Étatest toujours nécessaire et, dans l'affirmative comment cela peut-il se faire: la conjoncture économique, les difficultés de l'industrie touristique et la situation financière du gouvernement nous imposent une reformulation de ces deux questions.

Pour faire cet examen, il convient tout d'abord de rappeler, de façon factuelle, la place de l'industrie touristique dans l'ensemble de l'économie du Québec ainsi que son impact sur celle-ci.

Dans un deuxième temps, il apparaît important de dresser un tableau de l'intervention du ministère du Tourisme et plus particulièrement au chapitre des dépenses promotionnelles.

L'analyse de l'environnement du tourisme québécois constitue le troisième élément qu'il faut prendre en considération avant de répondre à la question concernant la nécessité de l'État.

À la suite de quoi, nous verrons la finalité d'une possible intervention de l'État, avant d'examiner les nouvelles modalités qui s'imposent désormais pour concevoir une telle intervention.

\section{Tourisme: une industrie essen- tielle à l'économie du Québec}

- 18000 entreprises québécoises ont réalisé en 1988, au seul chapitre du tourisme, 3,6 milliards de dollars, $11 \%$ de plus qu'en 1987.

- Àtitred'exemple, le ski alpin, avec un chiffre d'affaires de 500 millions, constitue une activité économique aussi importante que celle de grandes entreprises telles que Lavalin (525 millions), Cascade (588 millions) ou Culinar (518 millions).

- $\quad 38 \%$ de ces recettes sont réalisées par des entreprises impliquées dans l'hébergement et la restauration. Les entreprises de transport réalisent $24 \%$ dece chiffre d'affaires, les commerces de détail $23 \%$, tandis que le solde (15\%) revient à toutes sortes d'entreprises de services, celles des loisirs entre autres.

- En 1987, l'industrie touristique représentait $2,5 \%$ du produit intérieur brut de la province, ce qui est considérable, même si ce pourcentage dépassait les $3 \%$ en 1980 . Cette contribution au P.I.B. est supérieure à celle de l'industrie de l'habillement et voisine de celle d'une industrie aussi fondamentale que celle du matériel de transport.

- Le Québec réalise près de $41 \%$ de ses recettes touristiques avec des nonquébécois, avec des visiteurs venant des autres provinces canadiennes $(13,2 \%)$, des États-Unis $(18,6 \%)$ ou d'ailleurs dans le monde ( $9 \%$ ). L'industrie touristique, avec ses 980 millions de recettes réalisées avec des étrangers en 1988, se situe ainsi au $4 \mathrm{e}$ rang des industries exportatrices $\mathrm{du}$ Québec, derrière le papier d'impression, l'aluminium et le secteur automobile.

- Le Québec est néanmoins fortement deficitaire (671 millions de dollars en 1988) au chapitre du tourisme international puisque les dépenses des québécois à l'étranger ont atteint 1 milliard 651 millions de dollars en 1988 , le tourisme se situant ainsi au $2 \mathrm{e}$ rang des fuites de devises. 


\section{L'impact de l'industrie touristique}

- L'activité touristique québécoise génère 105000 emplois par année (90 000 emplois directs et 15000 emplois indirects). Mais le tourisme contribue, en fait, à l'existence de 250 000 emplois si l'on tient compte du caractère saisonnier de l'industrie et si l'on tient compte de son impact sur des activités reliées aux loisirs et aux services (postes d'essence, transport, etc.).

- L'industrie du tourisme est, en fait, une industrie intensive en maind'oeuvre puisque, pour une même production, le secteur touristique crée trois fois plus d'emplois que le secteur manufacturier.

- Chaque dollar de chiffre d'affaires en tourisme engendre des rentrées fiscales de 16 cents, dont 10 pour le gouvernement du Québec et 6 pour le gouvernement fédéral. Grâce au tourisme, les rentrées fiscales du Québec ont été de 355 millions de dollars en 1988 et celles du gouvernement fédéral ont été de 215 millions.

- Le tourisme constitue une activité économique essentielle pour les secteurs suivants:

les agences de voyages dont le chiffre d'affaires global a atteint 1,2 milliard de dollars en 1989;

les restaurants qui ont réalisé, en 1989 , un chiffre d' affaires d'environ 4,2 milliards de dollars (dont près de $50 \%$ par les restaurants détenant un permis d'alcool);

les hôtels qui, avec leurs 2000 établissements et leurs 70000 chambres, ont réalisé des ventes d'environ 1,3 milliard de dollars en 1989, au seul chapitre de l'hébergement leur chiffre d'affaires total s"établit à 3,5 milliards;

les pourvoiries (50 millions de dollars);

lescampings ( 65 millions de dollars); les congrès (200 millions de dollars).

\section{Le MTO: un ministère sans marge de manoeuvre}

- Avec un budget de 82,6 millions, le ministère du Tourisme ne dispose que de un quart de $1 \%$ des dépenses du gouvernement du Québec.

- $\quad 70 \%$ de ce budget vont à des dépenses que l'on peut qualifier de statutaires, difficilement compressibles: le financement de la dette et du déficit du Palais des Congrès $(25 \%)$, le fonctionnement de l'Institut du tourismeet de l'hôtellerie du Québec (21\%), et les frais de fonctionnement du Ministère ( $22 \%$ ) constitués des salaires des fonctionnaires, des frais de locations de bureau, des frais de fonctionnement du cabinet, etc. (voir tableau III).

- Il reste $30 \%$, soit au total 25 millions pourdoter les plans d'action du Ministère de moyens:

4 à 5 millions sont consacrés à l' accueil et aux renseignements des touristes;

4 a 5 millions contribuent de façon partielle, au développement des produits (ex: le jardin botanique, le biodome, etc.);

environ 12 millions sont consacrés à la promotion du tourisme intra Québec et hors Québec;

enfin, un peu plus de 4 millions sont redistribués aux 19 associations touristiques régionales.

- Les coupures imposées par le Conseil du trésor sur l'ensemble du budget du Ministère ne peuvent pratiquement pas s'appliquer aux dépenses statutaires si bien qu'elles seront concentrées sur les moyens d'action; la marge de manoeuve s'en trouve donc doublement réduite.

\section{Le budget promotionnel du Québec: I'écart se creuse avec les concurrents}

- De restriction en restriction, le budget consacré par le ministère du Tourisme a la promotion du Québec n'est plus que de 12 millions de dollars pour l'exercice 1989-1990, soit une baisse de $25 \%$ par rapport à l'année précédente.

- Le budget du premier concurrent du Québec, soit celui de l'Ontario est 2,2 fois plus élevé que celui du Québec (26,7 millions en 1989-1990).

- Ce décalageavec $1^{\dagger}$ Ontariose répercute d'année en année. Au cours des quatre dernières années, l'Ontario a consacré un total de 98 millions à la promotion du tourisme ontarien contre 54 millions de la part du Québec; le retard (sur quatre ans) est donc de 44 millions (voir Tableau I).

- Le Québec investit moins que la
Colombie-Britannique (18 millionsen 1989-1990) qui compte moins de trois millions d'habitants.

- Le Québec, qui est la dernière province du Canada quant a l'importance du tourisme dans son produit provincial brut, $n^{\prime}$ investit dans la promotion du tourisme que 1,8 dollar par habitant contre 3,0 dollars en Ontario, 4,5 dollars en Alberta et 6 dollars en Colombie-Britannique (voir Tableau II).

- Parallèlement, le gouvernement fédéral effectue lui aussi d'importantes coupures dans ses budgets promotionnels. Ceux-ci étaient de 35 millions en 1988-1989. Ils ne sont plus que de 30 millions pour l'exercice en cours et ne seront plus que de 25 millions en 1990-1991 et 20 millions en 1991-1992.

- Par ailleurs, le gouvernement fédéral n'entend pas renouveler les ententes federales-provinciales qui concernaient les grands événements, les congrès, la chasse et la pêche, sa présence dans les grands salons internationaux, etc.

- Les investissements effectués par les gouvernements municipaux ne viennent nullement pallier les réductions budgétaires des autres niveaux de gouvernement: pendant que la ville de Toronto consacre à sa promotion 8 millions sur les 12 millions du budget total consacré au tourisme et qu'Ottawa en consacre 3 sur un budget total de 5,1, Montréal ne dépense qu'un million en promotion sur un total de 3,4 millions au tourisme.

- La promotion du tourisme est pourtant le budget qui connaît au niveau international la plus forte croissance, les gouvernements étant non seulement convaincus de la rentabilité de cet investissement, mais conscients qu'il $s$ 'agit de l'arme indispensable pour se situer en bonne position au palmarès des pays gagnants de l'industrie qui sera la première au monde d' ici l'an 2000 (source: Office mondial du Tourisme). C'est ainsi que:

la France a triplé son budget promotionnel en 1989 par rapport à 1988 et prévoit l'accroître de $25 \%$ en 1990;

- Le Royaume-Uni consacre plus de 50 millions de dollars par année à la promotion touristique, 
- Les Êtats américains ont dépensé ensemble, près de 300 millions de dollars (canadiens) en 1988-1989 au chapitre du tourisme;

etc...

\section{L'environnement du tourisme québécois: des menaces sérieuses}

\section{1 - La TPS}

- A partirdu 1er janvier 1991, la taxe sur les produits et services, même à $7 \%$, sera néfaste à l'industrie touristique notamment dans le tourisme d'agrément; les gens d'affaires seront effectivement moins sensibles aux augmentations de prix étant donnéque la taxe payée sur leurs dépenses sera déduite des taxes perçues sur les ventes réalisées par leurs entreprises.

- La TPS, dans le secteur du tourisme, engendrera une hausse générale des prix d'à peu près $5 \%$, une hausse de beaucoup supérieure à l'inflation que cette taxe entraînerasur l'ensemble de l'économie ( $1 \%$ à $2 \%$ ) étant donné qu'en tourisme il ne s'agit que de services (et non pas de biens manufacturés) et que la valeur ajoutée y est très élevée.

- Cette hausse des prix freinera sensiblement les dépenses des consommateurs: selon l'Institut de la recherche en tourisme, la diminution de l'activité, au Québec, sera de 2,3\% dans le domaine de l'hébergement, de $1,3 \%$ dans la restauration et de $3,9 \%$ dans le transport aérien. Ces diminutions seront d'ailleurs beaucoup plus fortes au Québec qu'en Ontario étant donné que le Québec a une proportion tourisme-d'agrément versus tourisme-d'affaires supérieure à celle de $\mathrm{I}^{\prime}$ Ontario.

- Le Québec perdra, toujours selon I'IRT, 800 emplois à temps plein chaque année au cours de la période 1991-1993.

- Sur le plan fiscal, la TPS fera perdre 31 millions au trésor québécois à cause de la baisse d'activité du secteur. Les retombees fiscales du Québec passeront, sur la base de 1988 , de 355 millions à 324 millions. Pendant ce temps, le gouvernement fédéral qui a retiré (en 1988) 215 millions du tourisme québécois, en retirera désormais 395 millions, soit 180 millions de plus.
TABLEAU I

Ventillation du Budget - Ministere du Tourisme du Quebec Budget total (decembre 1989 ) $=82,6 \mathrm{M}$ \$

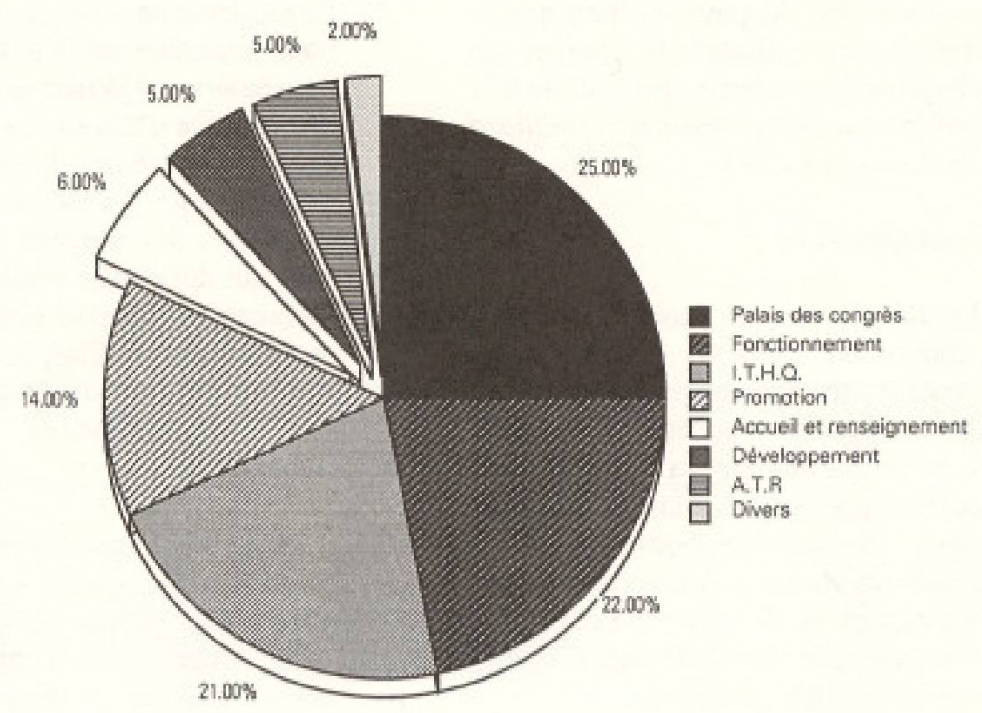

TABLEAU II

Promotion du tourisme - Evolution des budgets gouvernementaux len millions de \$)

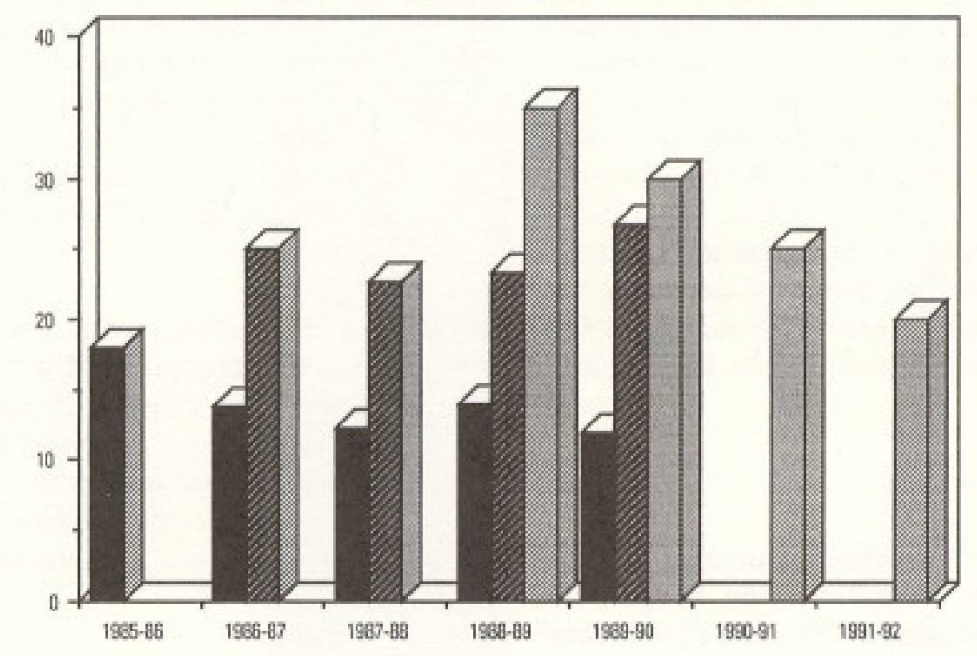

Source: Samson Bélair / Deloitte \& Touche

TABLEAU III

Investissements per capita consacrés à la promotion du tourisme (1989-1990)

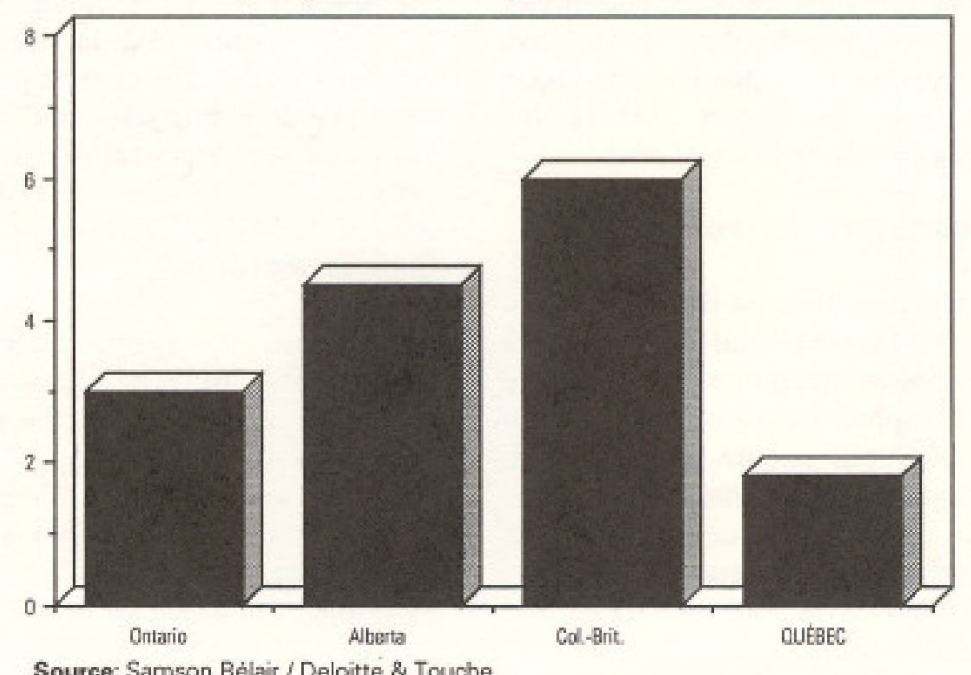

Source: Samson Belair / Deloitte \& Touche 
La TPS freinera la venue des visiteurs étrangers et incitera encore davantage les Québécois à passer leurs vacances à l'extérieur du pays; si bien que $l e$ déficit international du Québec au chapitre du tourisme, qui était de 671 millions en 1988, avoisinera le milliard de dollars en 1991 .

\section{2 - La conjoncture}

- Le ralentissement économique déjà amorcé en 1990 par la diminution gếnérale des activités en Amérique du Nord risque de s'accentuer, en 1991, avec non seulement la TPS, mais les inévitables coupures budgétaires des deux niveaux de gouvernements, compressions nécessairement drastiques et de surcroît couplées à une politique stricte de taux d'intérêt qui se veut déflationniste.

\section{3 - Le dollar canadien}

- Sa remontée jusqu'à 86 cents américains, a entraîné une diminution du nombre des touristes américains au Canada, et plus particulièrement en 1989. Le dollar est enfin à la baisse; mais même à 82 ou 83 cents, zone de fluctuation du dollar canadien au moment où ces lignes sont écrites, le dollar canadien est encore surévalué par rapport à sa vraie valeur qui devrait se situer quelque peu en bas de 80 cents.

\section{4 - Les coupures}

- Ce phénomène récurrent d'année en année au moment du budget, tend maintenant à s'installer également de façon imprévue à diverses reprises, au cours de l'exercice fiscal. Les gestionnaires, ceux de la fonction publique comme ceux des entreprises privées impliquées dans le tourisme, ne peuvent plus planifier de façon sérieuse, de façon fiable; les engagements ne tiennent plus.

\section{5 - La concurrence internationale}

- Le Québec, avec un demi de $1 \%$ du tourisme international doit faire face at une concurrence qui ne cesse de croître par la suppression des distances et qui s'accentuera encore à travers des changements fondamentaux tels que ceux d'Europe 92 ou à travers des événements d'envergure mondiale tels que les fêtes du 500e anniversaire de $1^{3}$ Amérique.
Le tourisme est un puzzle. Sans concertation, il n'existe pas

- Le tourisme ne se vend pas comme un bien manufacturé. En tourisme, un bien ne se vend jamais seul, quelle que soit sa force d'attrait. Le tourisme est toujours une chaîne d'éléments divers (une auberge, un lac, une spécificité régionale, des moyens de transport, etc.) qui doivent se vendre ensemble. Laconcertation estun impératif absolu en tourisme. L'État, les ATR et les associations sectorielles constituent ces indispensables agents de concertation.

- Le plan stratégique triennal, avec,pour horizon, 1992, que le Ministère s'est donné en 1988, constitue un exemple de cette concertation entre toutes les parties puisque le diagnostic et les solutions ont été énoncés lors de sessions publiques rassemblant tous les acteurs du Québec. Nombre de recommandations mises de l'avant par le rapport Samson Bélair/Deloitte \& Touche ont été mises en oeuvres au premier rang desquels l'approche "produits". Mais cette stratégie ne comporte qu'un seul défaut: une insuffisance de crédits pour lui donner l'élan mérité.

- La concertation, en fait, n"a de force que si elle est appuyée, d'une part, par de véritables moyens financiers et, d'autre part, par l'autorité d'un acteur capable de rassembler tout le monde, son autorité étant souvent reliée à l'importance des moyens financiers dont il dispose.

Or, au Québec, cette autorité revient au ministère du Tourisme; mais celui-ci manque cruellement de moyens, le budget consacré à la promotion devenant dérisoire face aux besoins et à la turbulence de l'environnement. Il n'est donc pas étonnant de voir perdurer les traditionnelles velleités individualistes: Montréal a longtemps offert cette image de tiraillement avec tous ses acteurs: le MTO, 1'OCTGM, le Palais des Congrès, les hôtels, etc.

Alors, face au bilan présenté précédemment, bilan imposant par le poids économique de l'industrie mais bilan inquiétant par la problêmatique budgétaire et par les menaces qui surgissent, et face à l'indispensable concertation imposée par la nature même du tourisme, le milieu va devoir réaliser qu'il doit se prendre en mains lui-même.
Les entreprises qui comprennent la nature mềme du tourisme et sa nécessaire concertation vont devoir s'associer pour assurer leur futur. Il faut prendre acte de la situation financière de l' État dont la marge de manoeuvre financièreira d'abord et avant tout à la santé et à l'éducation.

Cette prise en mains et cette concertation peuvent se concrétiser à travers la création d'une société d'économie mixte qui aura pour objet de promouvoir le tourisme québécois.

\section{Une proposition: la création d'une société d'économie mixte pour la promotion du tourisme québécois: la SPTQ}

Ladynamique de la révolution tranquille au Québec s'explique par la fantastique complicité qu'il y a eu et qu'il y a toujours - entre le secteur public et le secteur privé. Cette dynamique peut, plus que jamais, s'appliquer au tourisme par le biais d'une société d'économie mixte à laquelle les entreprises et l'État contribueraient chaque année dans le but d'assurer la promotion du tourisme tant à l'intra Québec que sur les marchés extérieurs. Il s'agirait ici de l'ensemble des activités reliées au marketing du tourisme québécois. Les contributions au budget de la SPTQ pourraient être les suivantes:

1- Le gouvernement du Québec consacre actuellement 25 millions $\$$ à ses plans d'actions (voir Tableau I) et un peu plus de 15 millions pour ses frais de fonctionnement. Si l'on retranche les dépenses consacrées au développement qui seraient requises par une réaffectation de ce voletà unorganisme tel que l'OPDQ, la contribution annuelle du gouvernement à la SPTQ pourrait être, sans augmentation de budget, de 30 millions \$.

2- Le milieu touristique, c'est-à-dire toutes les entreprises qui vivent du tourisme pourraient contribuer à la SPTQ par le biais d'une taxe, collectée par le gouvernement et reversée à la SPTQ, taxe minime variant de $1 \%$ à $2 \%$ selon unscénario qu'il conviendrait d'étudier beaucoup plus en profondeur, mais qui pourrait concerner les catégories suivantes:

- les agences de voyage factureraient à leurs clients une taxe de $2 \%$. Leur chiffre d'affaires étant réalisé essentiellement avec des Québécois voyageant hors Québec, cette 


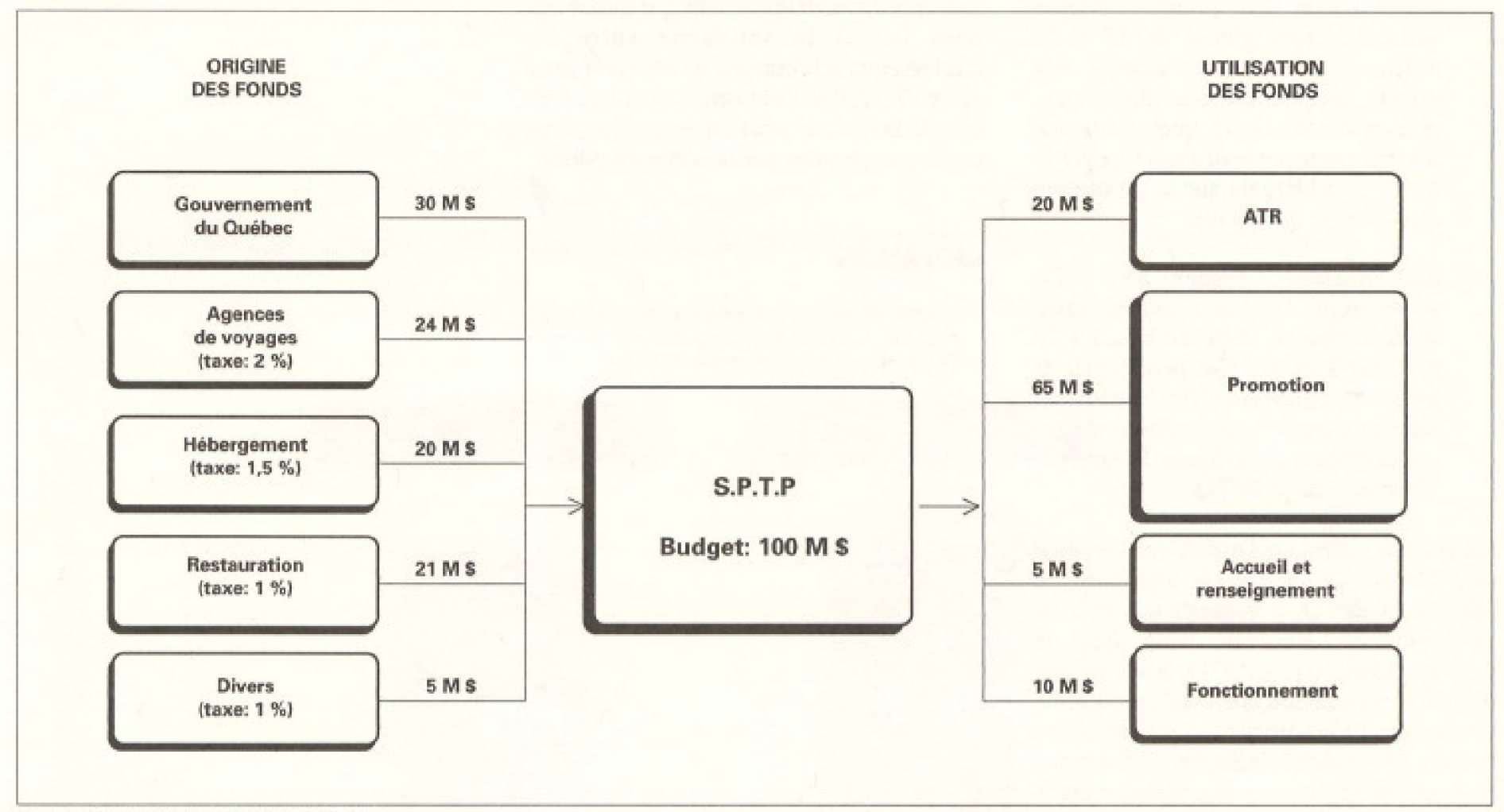

Source: Samson Bélair / Deloitte \& Touche

cotisation pourrait, un tout petit peu, endiguer le vaste mouvement de sorties de devises du Québec;

- pour l'industrie hôtelière, la taxe pourrait se situer autour de $1,5 \%$ en ce qui a trait à l'hébergement. Les hôtels sont les premiers bénéficiaires de la promotion touristique puisque leurs ventes dépendent considérablement du tourisme. Les hôtels, compte tenu de 1 'impact de la SPTQ, devraient considerer cette cotisation de $1,5 \%$ comme une contribution au marketing du Québec et des produits du Québec, contribution à déduire du total de leurs dépenses marketing. Celles-ci ont atteint environ 120 millions en 1989. Cette contribution ne devrait donc pas forcément se refléter dans le niveau des prix, contrairement à la TPS, qui est improductive, qui n'a rien à voiravec un investissement marketing. La contribution de 1 'industrie de l'hébergement à la SPTQ pourrait donc être de 20 millions \$/an;
- toutes les autres entreprises bénéficiant du tourisme pourraient contribuer à la SPTA à concurrence de $1 \%$ de leurs ventes. C'est ainsi que le seul secteur de la restauration, duquel il conviendrait de retirer tous les casse-crouttes, soit, de façon très globale, l'ensemble des restaurants ne disposant pas de permis d'alcool, pourrait contribuer à la SPTQ pour un montant de 21 millions \$ par année.

La formule de financement de la SPTQ. établie en fonction du chiffre d'affaires de l'ensemble du tourisme québécois, éviterait de tomber dans le dilemme de la "taxe de bienvenue "à $3 \%$ qui ne devrait s'appliquer qu'aux seuls hôtels alors que ses retombes bénéficieraient à d'autres intervenants de l'industrie touristique. Quant au budget de la SPTQ, évalué selon notre scénario à 100 millions $\$$, il pourrait être utilisé comme suit:

1- Fonctionnement. Un maximum de $10 \%$ du budget, soit 10 millions $\$$, devrait suffire pour supporter les frais des employés, les loyers, etc., d'autant plus que, concentrée à un seul endroit et n'ayant pas à vivre la complexité des organismes gouvernementaux (périodes électorales, changements d'organigrammes, surprises budgétaires, etc.), la Société d'économie mixte aurait tout lieu d'être productive;

2- Accueil et renseignement: Cette fonction est bien sûr essentielle et devrait être maintenue intégralement. Son budget actuel (frais de personnel inclus) est d'environ 5 millions $\$$;

3- La promotion, au Québec et surtout hors Québec, bénéficierait d' un budget de 65 millions \$. Il conviendrait sont simplement ici d'appliquer le plan stratégique du Ministère adopté fin 1988 qui, en plus de l'approche horizontaleclassique (positionnement général du Québec...) préconise une approche verticale, appelée également approche "produits". Madame Suzanne Chassé, dans Téoros, a clairement exposécette approche ainsi 
que les grands produits retenus par le Ministère (séjour, ski alpin, motoneige, congrès, etc.) $)^{1}$. C'est ainsi par exemple, que le seul produit " grandes villes", dont l'allocation budgétaire est d'environ $30 \%$, pourrait compter sur un budget global de 15 à 20 millions; ce montant, associé aux efforts supplémentaires du milieu, permet de réaliser à quel point une société mixte pourrait avoir un effet de levier considérable sur la promotion du tourisme québécois:

4- les sommes versées aux ATR pourraient, toujours selon notre scénario, passer de 4 millions $\$$ a 20 millions \$, ce qui leur permettrait de jouer beaucoup plus efficacement leur róle de rassembleur de l'offre au niveau régional tout en appliquant l'approche "produit", de la SPTQ.

Le gouvernement du Québec conserverait son rôle primordial dans le tourisme: au sein de la SPTQ, il jouerait son rổle de rassembleur d"autant plus que le gouvernement, par le MTO, a acquis une grande expérience en matière de promotion touristique. Le gouvemement pourrait aussi, comme partenaire de la Société d'économie mixte, s'assurer de la concordance entre la promotion et le développement fondamental des produits.

De fait, le gouvernement, par la création d'une direction "tourisme" au sein de l'OPDQ, continuerait à soutenir le développement des produits; cette intégration dans un organisme gouvernemental de développement très au fait des problématiques régionales permettrait

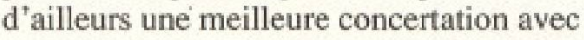
d'autres acteurs chargés du développement touristique étant donné que les grands produits ayant un impact touristique (ex: création d'un musée) ne relèvent que de façon infime du ministère du Tourisme.

\section{Conclusion}

La création d'une sociétéd'économie mixte pour la promotion du tourisme québécois nous semble une idée dont il conviendrait d'etudier plus à fonds la faisabilité. A priori, cette société donnerait de véritables moyens à l'industrie touristique; elle éviterait clairement une sorte de balkanisation du tourisme en rassemblant toute la profession et le gouvemement dans un seul organisme qui, par ses moyens financiers, exercerait le leadership indispensable dont le Québec a besoin pour affronter la conjoncture des années 1990.

Cette société mixte constituerait un autre exemple du modèle économique québécois. dans lequel la solidarité entre les intervenants et la complicité bien comprise entre l'ÉTAT et l'industrie constituent les clés de la réussite pour un petit pays quil ne craint pas de jouer sur la scène mondiale.

$+$

\footnotetext{
Note explicative

1. Chasse. Suranne, "Los vacances hivemales: une nouvelle approche marketing pour lo Oudbec". Teoros, Vol. 8, no. 3, novembre 1989, p.8
} 